\title{
Children's accuracy of portion size estimation using digital food images: effects of interface design and size of image on computer screen
}

\author{
Tom Baranowski ${ }^{1, *}$, Janice C Baranowski ${ }^{1}$, Kathleen B Watson ${ }^{1}$, Shelby Martin ${ }^{1}$, \\ Alicia Beltran ${ }^{1}$, Noemi Islam ${ }^{1}$, Hafza Dadabhoy ${ }^{1}$, Su-heyla Adame ${ }^{1}$, Karen Cullen ${ }^{1}$, \\ Debbe Thompson ${ }^{1}$, Richard Buday ${ }^{2}$ and Amy Subar ${ }^{3}$ \\ 'USDA/ARS Children's Nutrition Research Center, Department of Pediatrics, Baylor College of Medicine, 1100 \\ Bates Street, Houston, TX 77030-2600, USA: ${ }^{2}$ Archimage, Inc., Houston, TX, USA: ${ }^{3}$ National Cancer Institute, \\ Bethesda, MD, USA
}

Submitted 21 January 2010: Accepted 2 June 2010: First published online 15 November 2010

\begin{abstract}
Objective: To test the effect of image size and presence of size cues on the accuracy of portion size estimation by children.

Design: Children were randomly assigned to seeing images with or without food size cues (utensils and checked tablecloth) and were presented with sixteen food models (foods commonly eaten by children) in varying portion sizes, one at a time. They estimated each food model's portion size by selecting a digital food image. The same food images were presented in two ways: (i) as small, graduated portion size images all on one screen or (ii) by scrolling across large, graduated portion size images, one per sequential screen.

Setting: Laboratory-based with computer and food models.

Subjects: Volunteer multi-ethnic sample of 120 children, equally distributed by gender and ages (8 to 13 years) in 2008-2009.

Results: Average percentage of correctly classified foods was $60 \cdot 3 \%$. There were no differences in accuracy by any design factor or demographic characteristic. Multiple small pictures on the screen at once took half the time to estimate portion size compared with scrolling through large pictures. Larger pictures had more overestimation of size.

Conclusions: Multiple images of successively larger portion sizes of a food on one computer screen facilitated quicker portion size responses with no decrease in accuracy. This is the method of choice for portion size estimation on a computer.
\end{abstract}

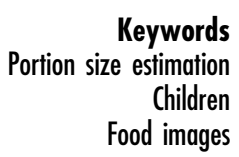

Since $24 \mathrm{~h}$ dietary recalls ( $24 \mathrm{hDR}$ ) have been identified as a useful method of diet assessment among children ${ }^{(1)}$, a self-administered computerized $24 \mathrm{hDR}$ program is being developed for children, called the Food Intake Recording Software System (FIRSSt) ${ }^{(2,3)}$. A key issue for dietary assessment is portion size estimation ${ }^{(4)}$. While there have been substantial problems in obtaining high portion size accuracy $^{(4)}$, use of food photographs has enhanced accuracy among adults ${ }^{(5)}$. A series of eight photographs in successively larger portions resulted in only $-4 \%$ to $+5 \%$ error in portion size estimation, compared with $-23 \%$ to $+9 \%$ using a single food photograph ${ }^{(5)}$. Small portion sizes tended to be overestimated while larger portion sizes tended to be underestimated ${ }^{(6)}$. When using computers to present visual images of foods, adults reported greater preference for multiple food images on one screen over successive presentations of various portion sizes ${ }^{(7)}$. Statistical research has estimated that validity coefficients of 0.9 or higher are needed to minimize misclassification error, which inhibits our ability to detect relationships with other variables or change from interventions ${ }^{(8)}$.

Screen images of foods also show promise for accurate portion size assessment among children. Among children aged 9-19 years, four photographs of successively larger portions led to $60 \%$ correct portion size selections ${ }^{(9)}$. It is possible that such food images could be so discrepant from actual that children would estimate too little (e.g. from small pictures) or too much (e.g. from large pictures).

*Corresponding author: Email tbaranow@bcm.tmc.edu

(C) The Authors 2010. The contribution by Amy Subar and Debbe Thomson is a work of the US Government and is not subject to copyright protection in the United States. 
This raises the question of the optimal size of images on the screen. The food portion images in one computerized program took only about one-ninth (or less) of the screen ${ }^{(7)}$. While selection from multiple images at a time is more efficient, how this size impacts the accuracy of portion size estimation for children is not known. Perceived size is likely a complex function of proximity of the image to the eye, the presence or absence of cues (i.e. fiducial markers) against which to check size, and the size of the image on the screen. As a result there were three research questions for the present study:

1. Does the size of picture (one-ninth of screen (method 1) $v$. whole screen (method 2)) influence the accuracy of estimation of portion size of foods of known size?

2. Do size cues (constant utensils and checked tablecloth $v$. no utensils or tablecloth) influence the accuracy of portion size estimation with pictures?

3. What size of image on the screen (e.g. $70 \%$ of real) best represents the size of that food, controlling for proximity of the eye and stable cues?

Based on cognitive information processing ${ }^{(10)}$, it was hypothesized that larger pictures (close to the size of the actual foods) would facilitate more accurate estimates of portion size and that the presence of image size cues would enhance accuracy, especially with the smaller pictures.

\section{Experimental methods}

\section{Design}

The study used an experimental design, balanced on food size cues group, gender (male/female) and age (8-13 years old). Diverse ages were used to test if there was an age at which children could not accurately and speedily conduct this task. Half the children were randomly assigned to seeing pictures with the size cues (see Fig. 1a) and half to the pictures without the size cues (see Fig. 1b). Children estimated the size of sixteen pre-specified food models twice. One time they completed the task with the larger pictures (see Fig. 1a) and one time with the smaller pictures (see Fig. 1b). To minimize an effect from fatigue, the presentation of the two tasks was randomly ordered.

The sixteen foods, the number of images and the size of the model for each food are identified in Table 1 . These foods were chosen because they are commonly consumed by children in this age range, as determined from the National Health and Nutrition Examination Survey (NHANES) database ${ }^{(11)}$. These foods also represented different types of image on the screen (e.g. distinct units, amorphous mounds, liquids in containers) which could influence ability to accurately estimate portion size. The portion sizes in the images were based on either standard item sizes defined in the Food and Nutrient Database for Dietary Studies (Food Survey Research Group, (a)

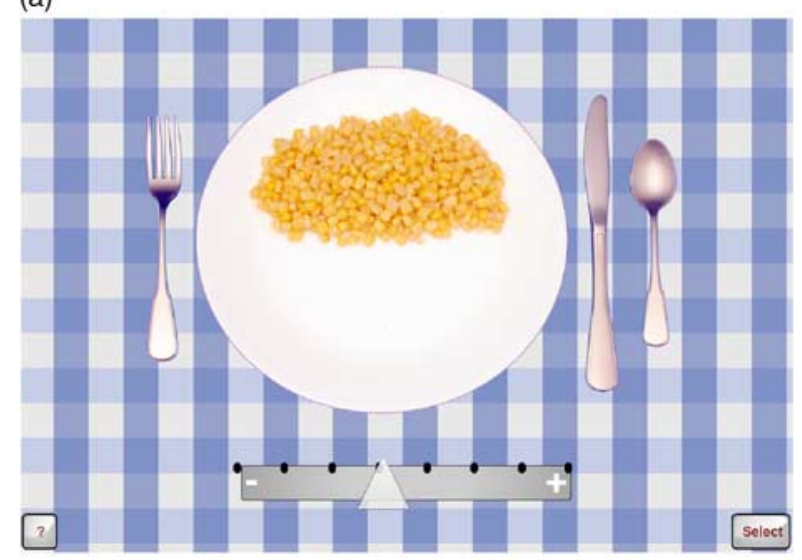

(b)

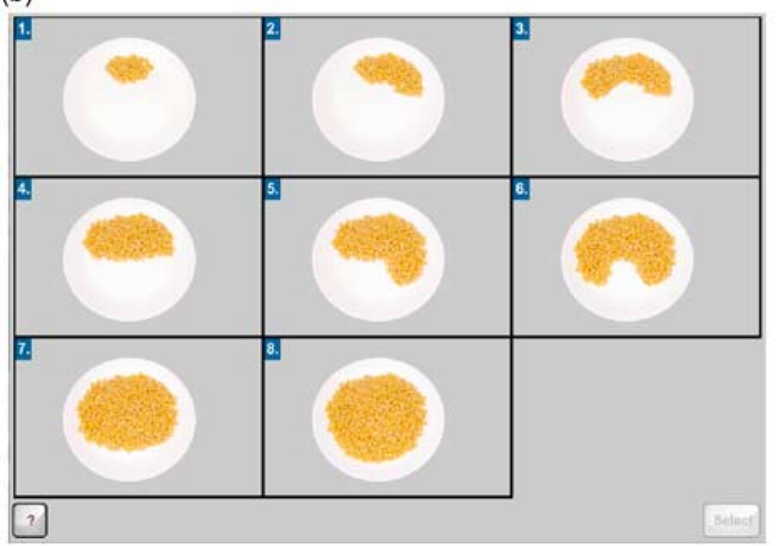

Fig. 1 Representative images of size of image on screen and presence of size cues: (a) large picture with food size cues; (b) small pictures without food size cues

Table 1 Foods in each task with the number and size of each food image and size of the food model in italics

A. Apple - three sizes: small, medium, large

B. Bagel - four sizes: mini, small, medium, large

C. Banana - three sizes: small, medium, large; four portions: $\frac{1}{4}, \frac{1}{2}, \frac{3}{4}$, 1 whole

D. Brownie - three sizes: small, medium, large

E. Cereal - eight sizes: $\frac{1}{4}$ cup, $\frac{1}{2}$ cup, $\frac{3}{4}$ cup, 1 cup, $1 \frac{1}{4}$ cups, $1 \frac{1}{2}$ cups, $1 \frac{3}{4}$ cups, 2 cups

F. Chocolate cake - three sizes: small, medium, large

G. Cola - four glass sizes: 6 fluid ounces, 12 fluid ounces short, 12 fluid ounces tall, 16 fluid ounces

H. Cookie - four sizes: small, medium, large, extra large

I. Corn - eight sizes: $\frac{1}{4}$ cup, $\frac{1}{2}$ cup, $\frac{3}{4}$ cup, 1 cup, $1 \frac{1}{4}$ cups, $1 \frac{1}{2}$ cups, $1 \frac{3}{4}$ cups, 2 cups

J. Fish fillet - four sizes: 1 ounce, 2 ounce, 3 ounce, 4 ounce

K. Grapes - eight sizes: $\frac{1}{4}$ cup, $\frac{1}{2}$ cup, $\frac{3}{4}$ cup, 1 cup, $1 \frac{1}{4}$ cups, $1 \frac{1}{2}$ cups, $1 \frac{3}{4}$ cups, 2 cups

L. Ketchup - three sizes: 1 tablespoon, 2 tablespoons, 3 tablespoons

M. Mashed potatoes - eight sizes: $\frac{1}{4}$ cup, $\frac{1}{2}$ cup, $\frac{3}{4}$ cup, 1 cup, $1 \frac{1}{4}$ cups, $1 \frac{1}{2}$ cups, $1 \frac{3}{4}$ cups, 2 cups

N. Pizza - two sizes: $1 / 16$ of 15 inch diameter, $1 / 8$ of 15 inch diameter

O. Soup - eight sizes: $\frac{1}{4}$ cup, $\frac{1}{2}$ cup, $\frac{3}{4}$ cup, 1 cup, $1 \frac{1}{4}$ cups,

$1 \frac{1}{2}$ cups, $1 \frac{3}{4}$ cups, 2 cups

P. Hamburger patty - three sizes: small, medium, large 
Agricultural Research Service, Beltsville, MD, USA) or percentiles of intake from NHANES 2003-2004 (from 5th to 95th percentile). To ensure consistent size across sessions and minimize problems of spoiling and shape distortion, the foods were presented to the child by realistic food models (Nasco, Fort Atkinson, WI, USA). Since this was a food portion size perception task (i.e. to determine if the children can correctly estimate the size of a food in front of them without error introduced by memory factors $)^{(10,12)}$, the plate, bowl or glass with the food model was placed right next to the keyboard (just to the left of the child for right-handed children or just to the right for left-handed children). The sequence of the sixteen foods within each task was randomly ordered by the computer. Some of these selections required a two-step process: (i) selection of the initial size of the container or item; and (ii) selection of the actual portion shown. For example, for banana the child first selected the size of the banana (small, medium, large) and then the amount of that size $\left(\frac{1}{4}, \frac{1}{2}, \frac{3}{4}\right.$, whole). To determine if liking or more frequently eating a food enabled the child to better report the portion of that food, each child was asked to rate their preference and how frequently they consumed each of the sixteen foods. A project staff person was present throughout the task to present the food model, ensure completion and facilitate problem-solving if something malfunctioned. To minimize influence of the staff person and to simulate what a computerized recall might do, a 'help menu' was created to provide strategic information at each point in the process and was activated by clicking on a 'help' button. At the completion of each of the two tasks the staff person asked the child questions about program preferences and ease of use.

Another task was presented to estimate the size on the screen that corresponded to perception of real size. In this task, there was a food image on the screen that corresponded to the food model. The child was asked to use a scroll bar on the right side of the screen to manipulate the size of the whole image (food size cues, tablecloth and food) from $50 \%$ of real to $150 \%$ of real. The child was asked to select the size of the image that directly corresponded to the size of the food model next to them. These foods were presented in random sequence and the initial size of the image on the screen was randomly selected, both by the computer.

\section{Study sample}

Children were included if they were: (i) between the ages of 8 and 13 years; (ii) able to speak English; (iii) able to read or answer questions; and (iv) did not have a physical, mental and/or visual limitation that would inhibit their ability to recall diet or use a computer. Children and their parents were recruited through the participant database of the Children's Nutrition Research Center (CNRC), by the center's recruiter. Families with eligible 8- to 13-year-old children, who previously indicated an interest in being contacted for studies, were identified and contacted. If they expressed interest, they were screened for inclusionary/exclusionary criteria. If they met the inclusionary criteria, they were scheduled for a visit. All data collection was completed at the CNRC in Houston, Texas. Baylor College of Medicine's Institutional Review Board approved the study. Parents signed informed consent and all children provided verbal assent. Twenty-five dollars was provided the child for participation.

\section{Measures}

The primary variables of interest were correspondence of the size of the food image on the screen to the size of the food model (correct, over- or underestimated) and time to select the image size. The computer program recorded these variables. The percentage of correct estimation was the percentage of foods for which the child selected the right image size; underestimated was the percentage of images selected that were smaller than the correct size; and overestimated was the percentage of images selected that were larger than the correct size. The time spent utilizing the 'help menu' was also collected and subtracted from the time spent for any foods invoking the help menu'. Aggregated child data were created by: (i) computing the percentage of the sixteen foods correctly sized by a child (0-100\%); and (ii) summing the times spent on all sixteen foods (excluding time spent with 'help'). The cola beverage was only used in method 2 and was excluded from the aggregated data.

To answer the third research question, the size of the food item on the computer screen relative to the size of the food model on the plate/bowl was recorded as a percentage of actual size (50\% to $150 \%$ ). An aggregated percentage of food sizes was obtained by averaging the mean percentage across foods for each child.

\section{Parent-completed information}

Parents completed a family demographic questionnaire, which included their child's status on medical conditions and medications, household membership and household socio-economic status, prior to beginning the study.

\section{Anthropometrics}

Trained and certified research staff used standardized protocols $^{(13)}$. Weight was measured twice using a model Alpha 882 scale from SECA Corporation (Hanover, MD, USA) and the two measurements averaged. Height was measured twice using a PE-AIM-101 stadiometer from Perspective Enterprises (Portage, MI, USA) and the two measurements averaged. BMI percentile was calculated with the Centers for Disease Control and Prevention (Atlanta, GA, USA) computerized program using the averaged height and weight measurements ${ }^{(14)}$. All children used a fixed standardheight chair and computer stand, and the computer's position was standardized on the computer stand to control for differences in the distance from the child's eyes to the 
screen. The child's seated height was measured using a freestanding sitting/standing height stadiometer (custom-made for the study by Prospective Enterprises), according to a standard protocol.

\section{Data analysis}

Multivariate analyses were used to investigate differences between methods (within-person factor) and interface designs (between-person factor). Level one analyses (aggregated by person) included two models (a) unadjusted and (b) adjusted for age, gender, race/ethnicity, adiposity index and highest level of household education. Model 1 (dependent variable: percentage of correctly sized foods) and Model 2 (dependent variable: time spent in program) utilized a multivariate analysis of variance model (MANOVA). Model 3 (dependent variable: percentage incorrect) utilized a doubly MANOVA model with two within factors (cues/no cues, percentage overestimation, percentage underestimation). All models included interaction terms with each covariate to determine if the covariate moderated any differences between methods. Level two analyses included the same series of models to investigate differences between methods and interface at the food level. Therefore, food was included as an additional within-person factor.

A doubly MANOVA was utilized for Model 4 (time). Due to the binary outcome, multivariate analyses with generalized estimating equations were used for Model 5 (percentage overestimation, percentage underestimation). Similar to level one, models included interaction terms with covariates. An additional adjustment to the model was made to determine whether food preference or frequency of consumption was associated with the timing or the sizing of the food items.

A univariate ANOVA was used to investigate differences between interface designs. Similar to analyses for the primary aims, Model 6 was (a) unadjusted and (b) adjusted for covariates.

\section{Results}

\section{Sample characteristics}

One hundred and twenty children participated. The sample was constrained to have equal numbers of children by interface design (food size cues $v$. no cues), gender and age (see Table 2). There were more white (36.7\%) and Hispanic (37.5\%) children than African-Americans (17.5\%) or other $(8.3 \%)$; more from households with degree level of education ( $57.5 \%)$; and more who were normal weight $(63.3 \%)$. There were no differences in these characteristics by interface design group.

\section{Main outcomes}

A simple ANOVA with the percentage of correctly sized foods as the dependent variable, with interface design
Table 2 Participant characteristics by study group: volunteer multiethnic children ( $n 120)$, equally distributed by gender and ages ( 8 to 13 years), 2008-2009

\begin{tabular}{|c|c|c|c|c|}
\hline \multirow[b]{2}{*}{ Characteristic } & \multicolumn{2}{|c|}{ Food size cues } & \multicolumn{2}{|c|}{ No food size cues } \\
\hline & $n$ & $\%$ & $n$ & $\%$ \\
\hline Total & 60 & $50 \cdot 0$ & 60 & $50 \cdot 0$ \\
\hline \multicolumn{5}{|l|}{ Gender } \\
\hline Female & 30 & $25 \cdot 0$ & 30 & $25 \cdot 0$ \\
\hline Male & 30 & $25 \cdot 0$ & 30 & $25 \cdot 0$ \\
\hline \multicolumn{5}{|l|}{ Age (years) } \\
\hline 8 & 10 & $8 \cdot 3$ & 10 & $8 \cdot 3$ \\
\hline 9 & 10 & $8 \cdot 3$ & 10 & $8 \cdot 3$ \\
\hline 10 & 10 & $8 \cdot 3$ & 10 & $8 \cdot 3$ \\
\hline 11 & 10 & $8 \cdot 3$ & 10 & $8 \cdot 3$ \\
\hline 12 & 10 & $8 \cdot 3$ & 10 & $8 \cdot 3$ \\
\hline 13 & 10 & $8 \cdot 3$ & 10 & $8 \cdot 3$ \\
\hline \multicolumn{5}{|l|}{ Race/ethnicity } \\
\hline White & 23 & $19 \cdot 2$ & 21 & $17 \cdot 5$ \\
\hline African-American & 9 & $7 \cdot 5$ & 12 & $10 \cdot 0$ \\
\hline Hispanic & 24 & $20 \cdot 0$ & 21 & $17 \cdot 5$ \\
\hline Other & 4 & $3 \cdot 3$ & 6 & $5 \cdot 0$ \\
\hline \multicolumn{5}{|l|}{ Household education } \\
\hline No degree & 24 & $20 \cdot 0$ & 27 & $22 \cdot 5$ \\
\hline Degree & 36 & $30 \cdot 0$ & 33 & $27 \cdot 5$ \\
\hline \multicolumn{5}{|l|}{ Adiposity index } \\
\hline Normal & 36 & $30 \cdot 0$ & 40 & $33 \cdot 3$ \\
\hline Overweight/obese & 24 & $20 \cdot 0$ & 20 & $16 \cdot 7$ \\
\hline
\end{tabular}

(size cues $v$. no cues), display method (small $v$. large pictures) and their interaction as factors, revealed no significant effects. Incorporating gender, race/ethnicity, household education, age, BMI, their two-way interactions with interface design and display method, and their three-way interactions with interface design by display method as factors also revealed no significant effects at $P \leq 0 \cdot 05$. The average percentage of correctly classified foods across display methods and interface design was $60 \cdot 3 \%$ (sD 12.6\%).

The same simple ANOVA with total time spent estimating portion size as the dependent variable revealed a main effect only for display method $(P \leq 0 \cdot 000)$, with multiple smaller pictures on the screen taking less time (mean $2.8(\mathrm{SD} 0 \cdot 7) \mathrm{min}$ ) than one larger picture on the screen (mean $4.4(\mathrm{SD} 1.4) \mathrm{min}$ ). Incorporating all the other variables into the model left this single main effect and added no others.

\section{Percentage over- and underestimated}

The simple doubly multivariate analysis of percentage of foods that were overestimated or underestimated revealed a significant $(P \leq 0 \cdot 000)$ main effect for type and a method-by-type interaction term $(P \leq 0 \cdot 000)$. Controlling for demographic characteristics and BMI did not change the type main effect $(P \leq 0 \cdot 002)$ or the methodby-type interaction $(P \leq 0 \cdot 000)$, but also revealed a significant type-by-BMI effect $(P \leq 0 \cdot 006)$, as presented in Fig. 2. There was substantial convergence in over- and underestimation with the small pictures for both BMI groups, but substantial divergence with large pictures. 
There was more overestimation with the larger pictures. There was a convergence of over- and under-reporting among the overweight and obese (Fig. 2).

\section{Analyses by foods}

Differences were detected in the simple multivariate analysis in the percentage of correctly sized across foods and a method-by-food interaction $(P \leq 0 \cdot 000)$, which did not change when controlling for the demographic variables and BMI $(P \leq 0 \cdot 003)$. No additional significant effects were detected in the latter model. The percentage correct for each food item by small or large pictures is depicted in Fig. 3. For some foods the larger pictures led to more accurate estimation of portion size while the smaller pictures were more accurate for other foods. The differences were statistically significantly different for grapes, hamburger patty and brownie.

Differences were detected in the simple doubly multivariate model in amount of time used to match foods (food main effect; $P \leq 0 \cdot 000$ ) and a method-by-food interaction $(P \leq 0 \cdot 000)$, which did not change when controlling for the demographic variables and BMI. No other significant effects were detected in the latter model. It took more time to report the size of the larger pictures, most likely due to the necessary scrolling (Fig. 4).

\section{Size of picture on screen}

The simple ANOVA on average perceived size of food on the screen revealed no significant effect for interface design. Entering the demographic characteristics and BMI and the demographics-by-interface-design interaction term revealed a significant gender effect $(P<0 \cdot 015)$. When the individual foods were included in a repeatedmeasures design, the model revealed significant differences across foods $(P<0 \cdot 000)$, by gender $(P<0 \cdot 013$ as reported above) and a food-by-interface-by-BMI interaction ( $P \leq 0 \cdot 015$; Fig. 5).

Child seated height was highly significantly bivariately correlated with age (Pearson $r=0 \cdot 82, P<0 \cdot 000$ ), significantly correlated with percentage of foods correctly classified by larger pictures $(r=0 \cdot 20, P \leq 0.03)$ and smaller pictures $(r=0 \cdot 24, P \leq 0 \cdot 009)$, but not significantly

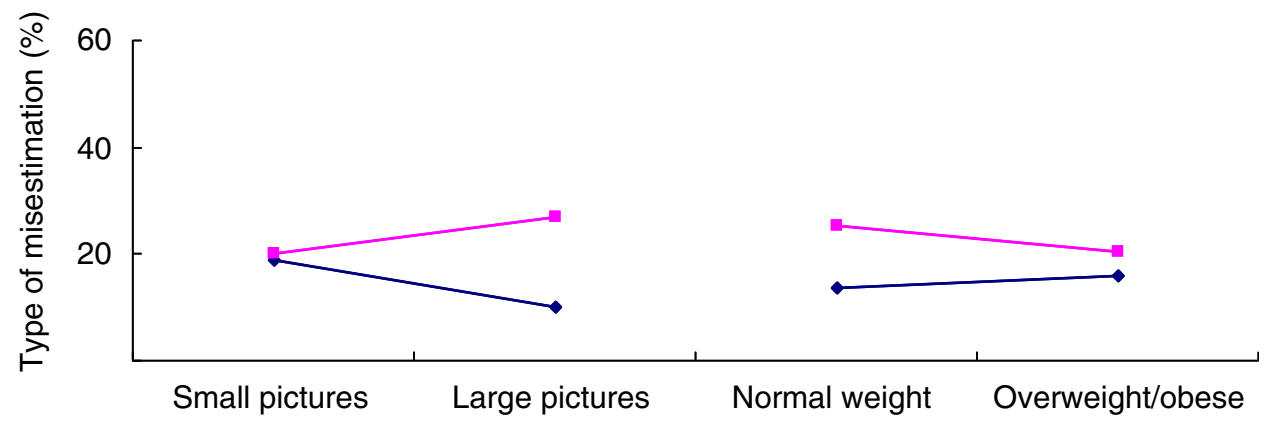

Fig. 2 Percentage of underestimation (- -) and overestimation ( $-\mathbf{-}-$ ) of portion size by display method (small picture, large picture) and BMI among volunteer multi-ethnic children ( $n$ 120), equally distributed by gender and ages (8 to 13 years), $2008-2009$

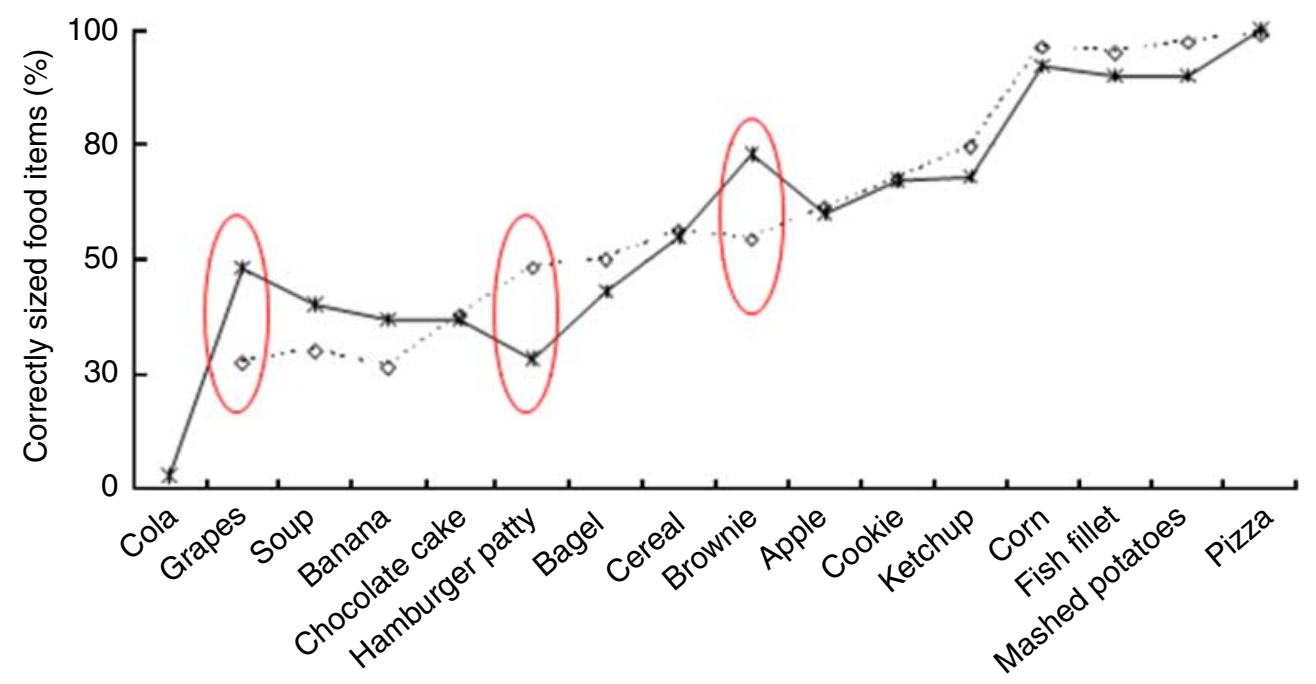

Fig. 3 Percentage of correctly sized food items by display method (- - --- , small images; $-*-*$, large images) among volunteer multi-ethnic children ( $n$ 120), equally distributed by gender and ages (8 to 13 years), 2008-2009 


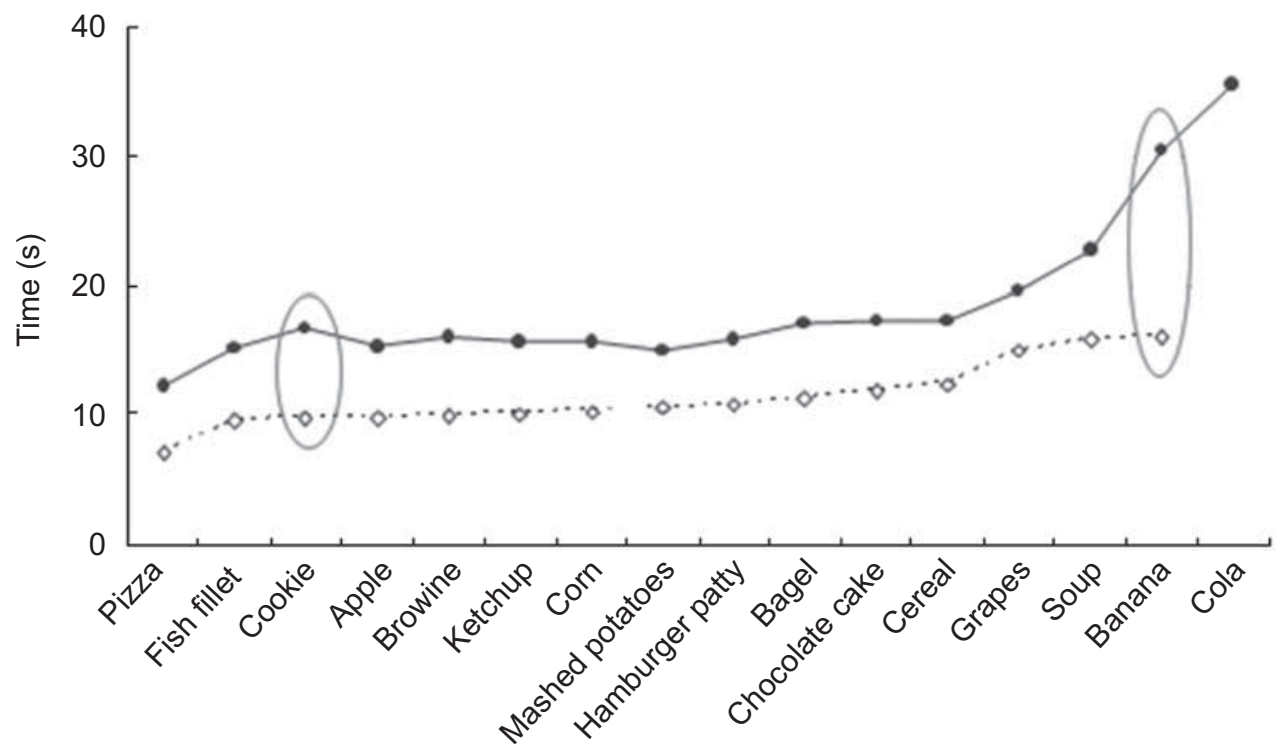

Fig. 4 Time (in seconds) for reporting size of food items by display method (- - $\diamond--$, small images; $-\diamond-$, large images) among volunteer multi-ethnic children ( $n$ 120), equally distributed by gender and ages ( 8 to 13 years), 2008-2009

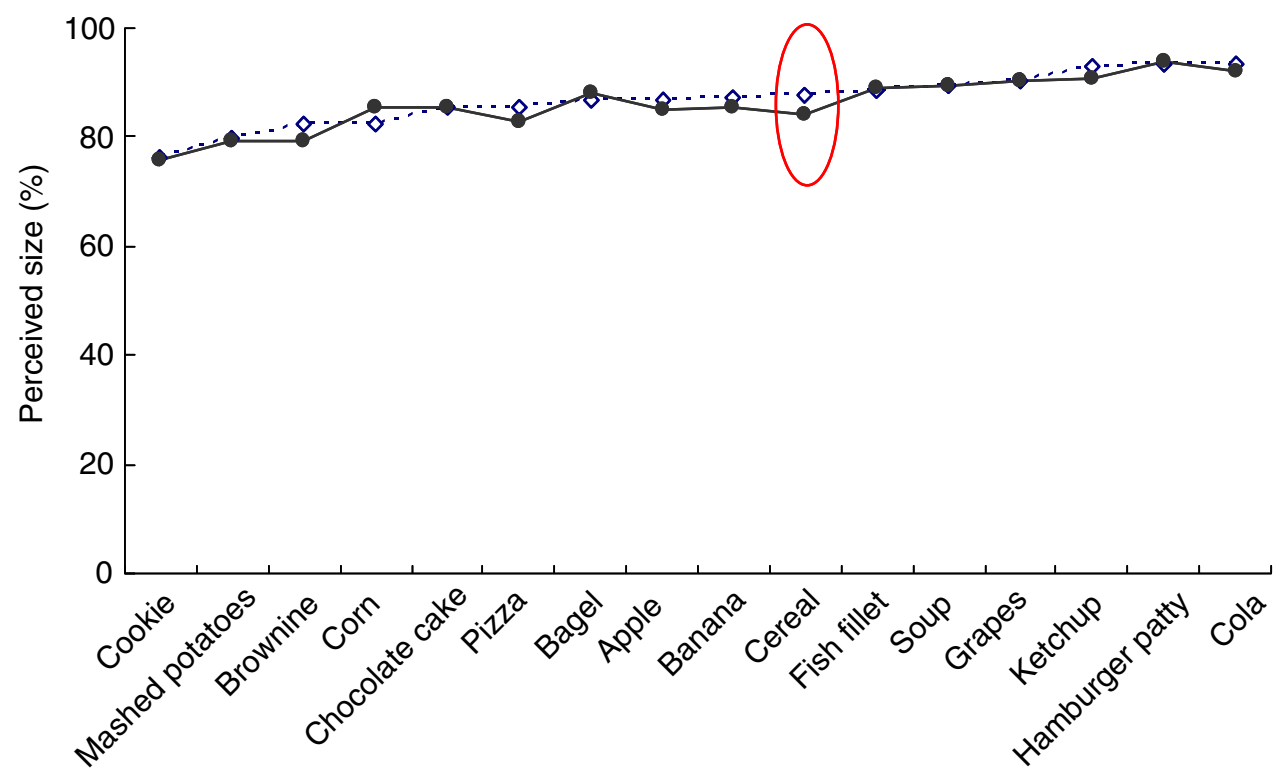

Fig. 5 Percentage of perceived size for food items by interface design (-•-, food size cues; - - $\diamond--$, no food size cues) among volunteer multi-ethnic children ( $n$ 120), equally distributed by gender and ages ( 8 to 13 years), 2008-2009

related to total time to complete method 1 or 2 or to percentage of under- or overestimation for either method. When seated height was centred and added to all the models, the results remained nearly unchanged.

Analysis of semi-qualitative data revealed that just over $40 \%$ of children preferred each size picture: those who preferred the larger pictures did so because it was easier to compare to the food model, but those who liked smaller pictures was because it was easier to make a choice. Most thought it was very easy $(43 \cdot 3 \%)$ or a little easy $(42 \cdot 5 \%)$ to find the picture that best matched the food model.

\section{Discussion}

Correct classification of foods using images was $60 \cdot 3 \%$. This is similar to the $60 \%$ reported among 9- to 19-year-olds using four pictures ${ }^{(9)}$. Different measures of agreement precluded comparisons across studies. With regard to the first two research questions, no differences in accuracy were detected by either design feature. We found more overestimation with larger images, and about equal overand underestimation with the multiple smaller images on the screen. Children elsewhere have been found to more 
likely overestimate in general (e.g. by $46 \%{ }^{(15)}, 0-142 \%{ }^{(9)}$ ). In contrast to the existing literature on under-reporting of total energy by the obese ${ }^{(16)}$, the overweight/obese were less likely to overestimate portion size than normal-weight children. Thus under-reporting of total energy intake does not appear to be due to under-reporting of portion size.

Statistically significant differences in accuracy of size categorization between small and large pictures were obtained for grapes (large pictures more likely to be correct), hamburger patty (small pictures more likely to be correct) and brownie (larger pictures more likely to be correct). The high accuracy for size categorization of pizza was likely due to having pictures of only two servings on the screen. However, high accuracy was also obtained for corn (eight image sizes), fish fillet (four image sizes) and mashed potatoes (eight image sizes), which had many more sizes of image. None of the manipulations in the present study appeared to enhance accuracy. Further research is needed on other manipulations to increase accuracy of report of portion size beyond $60 \cdot 3 \%$.

Substantially less time was taken to classify foods using the smaller, rather than the larger pictures, with no differences in accuracy detected. Less time was likely due to children not having to scroll across pictures and greater ease in making perceptual equivalence relationships when all possibilities were on the screens. This suggests that multiple small pictures on the screen at the same time is the preferred method for a computerized $24 \mathrm{hDR}$ for children, similar to the findings among adults ${ }^{(7)}$.

Regarding research question three, the average size of the image on the screen was $83 \%$ of actual, ranging from $76 \cdot 1 \%$ for a cookie to $93.7 \%$ for the hamburger patty with no systematic difference by food size cues. We have seen no report of percentage size for images on screen in the literature.

There was no evidence that the younger children in this age range ( $8-13$ years) were any less accurate or took more time to select the picture sizes, suggesting that the pictures levelled the playing field among younger children who might have difficulties with food category placements ${ }^{(3)}$. No age-related differences in accuracy were reported in other studies $^{(9,12,15)}$. No significant differences in accuracy or time were detected by gender, similar to other studies ${ }^{(17)}$.

The strengths of the current study include a reasonably large multi-ethnic sample of boys and girls, and controlling for several possible confounding variables. While the food images in this research should have reasonably corresponded to the size of the food models, future research may benefit from attention to child-specific portion sizes ${ }^{(15)}$.

\section{Conclusions}

Multiple small food pictures on the screen at the same time decreased the time needed to make size judgements with no diminution in accuracy. Thus, multiple small pictures appear to be the preferred method. Further research is needed to identify methods that enhance children's accuracy of report of portion size beyond $60 \cdot 3 \%$ to minimize misclassification bias in the future.

\section{Acknowledgements}

Sources of funding: This research was funded primarily by a grant from the National Cancer Institute (5 U01 CA13076202). This work is also a publication of the United States Department of Agriculture/Agricultural Research Service (USDA/ARS) Children's Nutrition Research Center, Department of Pediatrics, Baylor College of Medicine, Houston, Texas, and has been funded in part with federal funds from the USDA/ARS under Cooperative Agreement No. 58-62506001. The contents of this publication do not necessarily reflect the views or policies of the USDA, nor does mention of trade names, commercial products or organizations imply endorsement from the US government. Conflicts of interest: There are no conflicts of interest. Author contributions: T.B. conceived the study, secured the funding and wrote a first draft. J.C.B. managed the conduct of the study. K.B.W. conducted all the analyses. S.M. managed the staff and coding of all comments. N.I. contributed to the design of the study, H.D. and S.A. took the pictures, conducted beta testing of the program and collected the data. K.C. and D.T. contributed to the design of the study; D.T. trained for qualitative data collection skills. R.B. did the programming. A.S. discussed concepts and critically reviewed drafts of the design and methods of the study, and critically reviewed drafts of the manuscript. All co-authors reviewed and critiqued multiple drafts of the manuscripts.

\section{References}

1. McPherson RS, Hoelsher DM, Alexander M et al. (2000) Dietary assessment methods among school-aged children: validity and reliability. Prev Med 31, Suppl. 2, S11-S33.

2. Baranowski T, Islam N, Baranowski J et al. (2002) The food intake recording software system is valid among fourthgrade children. J Am Diet Assoc 102, 380-385.

3. Baranowski T, Beltran A, Martin S et al. (2010) Tests of the accuracy and speed of categorizing foods into child vs professional categories using two methods of browsing with children. J Am Diet Assoc 110, 91-94.

4. Cypel YS, Guenther PM \& Petot GJ (1997) Validity of portion-size measurement aids: a review. J Am Diet Assoc 97, 289-292.

5. Nelson M, Atkinson M \& Darbyshire S (1994) Food photography. I: The perception of food portion size from photographs. BrJ Nutr 72, 649-663.

6. Nelson M, Atkinson M \& Darbyshire S (1996) Food photography. II: Use of food photographs for estimating portion size and the nutrient content of meals. Br J Nutr 76, 31-49.

7. Subar A, Crafts J, Zimmerman T et al. (2010) Assessment of the accuracy of portion size reports using computer-based food photography aids in the development of an automated self-administered 24-hour recall. J Am Diet Assoc 110, 55-64.

8. De Moor C, Baranowski T, Cullen KW et al. (2003) Misclassification associated with measurement error in the 
assessment of dietary intake. Public Health Nutr 6, 393-399.

9. Lillegaard IT, Overby NC \& Andersen LF (2005) Can children and adolescents use photographs of food to estimate portion sizes? Eur J Clin Nutr 59, 611-617.

10. Baranowski T \& Domel SB (1994) A cognitive model of children's reporting of food intake. Am J Clin Nutr 59, 1 Suppl., 212S-217S.

11. Dave JM, Evans AE, Saunders RP et al. (2009) Associations among food insecurity, acculturation, demographic factors, and fruit and vegetable intake at home in Hispanic children. J Am Diet Assoc 109, 697-701.

12. Foster E, O'Keeffe M, Matthews JN et al. (2008) Children's estimates of food portion size: the effect of timing of dietary interview on the accuracy of children's portion size estimates. Br J Nutr 99, 185-190.
13. Lohman TG, Roche AF \& Martorell R (1988) Anthropometric Standardization Reference Manual. Champaign, IL: Human Kinetics Books.

14. Kuczmarski RJ, Ogden CL, Guo SS et al. (2002) 2000 CDC Growth Charts for the United States: methods and development. Vital Health Stat 11 issue 246, 1-190.

15. Foster E, Matthews J, Nelson M et al. (2005) Accuracy of estimates of food portion size using food photographs the importance of using age-appropriate tools. Public Health Nutr 9, 509-514.

16. Livingstone $\mathrm{MB}$ \& Black AE (2003) Markers of the validity of reported energy intake. J Nutr 133, Suppl. 3, 895S-920S.

17. Steyn NP, Senekal M, Norris SA et al. (2006) How well do adolescents determine portion sizes of foods and beverages? Asia Pac J Clin Nutr 15, 35-42. 